\title{
Avaliação da ferida e cuidados do enfermeiro em pacientes diabéticos portadores de úlcera venosa
}

\author{
Wound assessment and nursing care in diabetic patients with venous ulcers
}

Evaluación de heridas y cuidados de enfermería en pacientes diabéticos con úlceras venosas

Rose Valda de Andrade ${ }^{1 *}$, Luana Dias de Alencar Lima de Almeida ${ }^{1}$, Roberta de Melo Galdino ${ }^{1}$, Elanny Santana Brito ${ }^{1}$, Rafaela Nacimento Ribeiro ${ }^{1}$, Mirthis Sento-Sé Pimentel Magalhães ${ }^{1}$, Josilma Granja Costa $^{1}$, Márcia Sento Sé Magalhães Pimentel ${ }^{2}$.

\section{RESUMO}

Objetivo: Descrever o cuidado do enfermeiro à pacientes portadores de Diabetes com úlcera venosa. Métodos: Trata-se de uma pesquisa de campo, parecer do comitê de ética, oㅜ 3.666.833 com caráter descritivo, analítico, de abordagem qualitativa que foi desenvolvido no Instituto de Saúde de um Centro Universitário de Salvador/BA. A coleta de dados foi realizada através de análise de prontuários no período de abril a setembro de 2019 , onde de 40 pacientes diabéticos, foram selecionados apenas 05 , sendo estes diabéticos e portadores de úlcera venosa. Resultados: Foi identificada que a relação do diabetes com a úlcera venosa se dá pela dificuldade do processo cicatricial que o paciente acometido com DM desenvolve, gerando em muitos casos depressão e baixa autoestima. Conclusão: Conclui-se que, as úlceras venosas têm uma maior dificuldade de cicatrização aos pacientes diabéticos e principalmente os que apresentam descompensação da doença. Assim, é necessário que o enfermeiro amplie seus conhecimentos, podendo ir além de simples estratégias ou cuidados de conforto, mas realizando intervenção que busque a resolução de um problema baseado em entendimento, promovendo um tratamento ideal para o paciente.

Palavras-chave: Úlcera varicosa, Diabetes mellitus, Enfermeiros.

\begin{abstract}
Objective: To describe the nurse's care to Diabetes patients with venous ulcers. Methods: This is a field research, opinion of the ethics committee, No. 3,666,833 with a descriptive, analytical character, with a qualitative approach that was developed at the Health Institute of a University Center in Salvador / BA. Data collection was performed through analysis of medical records from April to September 2019, where out of 40 diabetic patients, only 5 were selected, being these diabetics and patients with venous ulcers. Results: It was identified that the relationship between diabetes and venous ulcers is due to the difficulty of the healing process that the patient with DM develops, generating in many cases depression and low self-esteem. Conclusion: It is concluded that, venous ulcers have greater difficulty in healing patients with diabetes and especially those who present decompensation of the disease. Thus, it is necessary for nurses to expand their knowledge, being able to go beyond simple strategies or comfort care but performing an intervention that seeks to solve a problem based on understanding, promoting an ideal treatment for the patient.
\end{abstract}

Keywords: Varicose ulcer, Diabetes mellitus, Nurses.

${ }_{1}^{1}$ Centro Universitário Jorge Amado. Salvador - BA. *E-mail: roziandrade30@hotmail.com

${ }^{2}$ Hospital Aliança. Salvador - BA.

SUBMETIDO EM: 2/2020 ｜ ACEITO EM: 2/2020 ｜ PUBLICADO EM: 5/2020

REAS/EJCH | Vol.Sup.n.48 | e3070 | DOI: https://doi.org/10.25248/reas.e3070.2020 Página 1 de 8 


\section{RESUMEN}

Objetivo: Describir la atención de la enfermera a pacientes con diabetes con úlceras venosas. Métodos: Esta es una investigación de campo, opinión del comité de ética, No. 3,666,833 con un carácter descriptivo, analítico, con un enfoque cualitativo que se desarrolló en el Instituto de Salud de un Centro Universitario en Salvador / BA. La recopilación de datos se realizó a través del análisis de registros médicos de abril a septiembre de 2019, donde de 40 pacientes diabéticos, solo se seleccionaron 5 , siendo estos diabéticos y pacientes con úlceras venosas. Resultados: Se identificó que la relación entre diabetes y úlceras venosas se debe a la dificultad del proceso de curación que desarrolla el paciente con DM, lo que genera en muchos casos depresión y baja autoestima. Conclusión: Se concluye que las úlceras venosas tienen una mayor dificultad para curar a pacientes con diabetes y especialmente a aquellos que tienen enfermedad descompensada. Por lo tanto, es necesario que las enfermeras amplíen sus conocimientos, puedan ir más allá de las estrategias simples o el cuidado de la comodidad, pero realicen una intervención que busque resolver un problema basado en la comprensión, promoviendo un tratamiento ideal para el paciente.

Palabras clave: Úlcera varicosa, Diabetes mellitus, Enfermeiras.

\section{INTRODUÇÃO}

Diabetes Mellitus (DM) é considerada uma Doença Crônica não Transmissível (DCNT), decorrente da ação da insulina ou de sua incapacidade de exercer sua função, comprometendo o metabolismo dos lipídios, carboidratos e proteínas do organismo. Assim, pode ser classificado em tipo 1 A e B e tipo 2. O tipo 1 A é conhecido também como autoimune, ocorrendo autodestruição das células beta pancreáticas, levando a incompetência do organismo na produção de insulina e o tipo $1 \mathrm{~B}$, é de causa desconhecida. $\mathrm{O}$ tipo 2, mais predominante em adultos e o tipo de maior incidência, é resultante da produção diminuída ou da resistência à insulina (SOCIEDADE BRASILEIRA DE DIABETES, 2018).

No Brasil, há uma estimativa que $3 \%$ da população possuem ferida, visto que indivíduos portadores de DM dispõem a apresentar uma maior probalilidade de desenvolver lesões, propiciando uma elevação do número de ocorrências (LEAL TS, et al., 2017).

A Úlcera Venosa (UV) é uma lesão crônica que normalmente surge em região tibial e maleolar. Sua etiologia é resultante da Insuficiência Venosa Crônica (IVC) devido a uma osbtrução ou incapacidade das válvulas do sistema venoso, ocasionando o refluxo ou incompetência na bomba do músculo gastrocnêmio (DIAS TYAF, et al., 2014). Esse tipo de úlcera tem alta taxa de recidiva, a terapêutica é complexa e a ferida ocasiona sofrimento e danos à qualidade de vida do paciente (SALVETTI MG, et al., 2014).

Alguns aspectos de risco predispõem o indivíduo para o desenvolvimento da insuficiência venosa crônica como: hereditariedade, multiparidade, obesidade, trombose venosa profunda, DM, Hipertensão Arterial Sistêmica (HAS) (BORGES L, et al., 2016).

Desse modo, o papel do enfermeiro é de fundamental importância à assistência desses pacientes, principalmente na escolha da terapia, que deve ser baseada no conhecimento científico e no olhar holístico, com a finalidade de proporcionar a recuperação e reabilitação do paciente, assim como dos familiares que devem ser envolvidos no processo do cuidado (JOAQUIM FL, et al., 2018).

É necessário que o enfermeiro compreenda todos os passos do processo de reparação tecidual, para que possa prestar uma assistência de qualidade e individual, pois cada paciente apresenta uma característica diferente. Além disso, é necessário identificar as doenças de base e suas implicações, além de conhecer as características clínicas e histopatológicas das úlceras a fim de direcionar a assistência (SOUZA LJ, et al., 2014).

REAS/EJCH | Vol.Sup.n.48 | e3070 | DOI: https://doi.org/10.25248/reas.e3070.2020 Página 2 de 8 
Assim, tornou-se relevante o desenvolvimento desse estudo devido à necessidade de conhecer as limitações e as estratégias de enfrentamento das pessoas acometidas por ferida crônica, além de contribuir para uma melhor compreensão do profissional de saúde perante a assistência. É importante ressaltar, que os resultados do estudo poderão servir de referência para a formação de novos conhecimentos acerca da temática. Qual o critério de avaliação do enfermeiro diante de pacientes portadores de diabetes com úlcera venosa? Contudo, o objetivo do nosso trabalho é descrever a importância da avaliação da ferida e ações aplicadas ao cuidado do enfermeiro em pacientes portadores de úlcera venosa.

\section{MÉTODOS}

Trata-se de uma pesquisa de campo de caráter descritivo, analítico e abordagem qualitativa que foi realizada em coletas de prontuários de pacientes portadores de DM que desenvolveram úlcera venosa crônica, e que são atendidos no institudo de saúde do centro de Feridas de uma Instituição de Ensino privada de Salvador/BA.

A pesquisa foi realizada no período de abril a setembro de 2019, onde foram avaliados 40 prontuários, sendo selecionados apenas 5. Foram utilizados os critérios de inclusão como: paciente ser diabético e portador de UV. Os critérios de exclusão adotados foram os pacientes que não possuem lesão em membros inferiores de etiologia venosa e que não possuam DM.

Os dados foram coletados através de uma ficha de avaliação, com perguntas que abordam o histórico do paciente, antecedentes pessoais, antecedentes familiares e hábitos de vida, assim como a avaliação e evolução da lesão, com análise do registro dos prontuários da etiologia da lesão, tratamento anterior que o paciente foi submetido, tratamento atual, localização da lesão e avaliação da lesão.

A ficha de avaliação foi estruturada contendo questionamentos que abordavam variáveis sociodemográficas (sexo, idade, escolaridade, estado civil, cor/raça, profissão, endereço); variáveis de saúde (peso, altura, índice de massa corpórea- IMC, antecedentes pessoais, antecedentes familiares, hábitos de vida); e variáveis relativas à história e ao tratamento da ferida (etiologia da lesão, tratamentos anteriores da lesão, tratamento atual, localização da lesão, avaliação dos registros de evolução da lesão e características da lesão, como leito da ferida, características do exsudato, bordas e área perilesional).

Esta pesquisa foi aprovada pelo Comitê de Ética em Pesquisa (CEP) do Instituto de Ensino Superior da Bahia IMES, através do Certificado de Apresentação para Apreciação Ética (CAAE), no 23531919.0.0000.5032, parecer no 3.666.833. estando este de acordo com o que preconiza a Resolução 466/2012, do Conselho Nacional de Saúde com pesquisas envolvendo seres humanos.

\section{RESULTADOS E DISCUSSÃO}

Após a análise dos 5 prontuários de pacientes que obedeciam os critérios de inclusão do estudo. A amostra foi composta por indivíduos com idade mínima de 45 anos e a máxima de 74 anos. A prevalência foi de indivíduos do sexo masculino e que possuiam grau de escolaridade $2^{\circ}$ grau completo é importante estar atento ao grau de instrução do sujeito atendido, pois a baixa escolaridade pode interferir diretamente na compreensão da patologia,na identificação da técnica de cuidado, na aplicação das intervenções relacionadas ao tratamento das lesões e alteração da conduta e costume no campo domiciliar (JOAQUIM FL, et al., 2017).

Os critérios de inclusão foram: pacientes serem acometidos por DM e que estejam em tratamento da ferida de etiologia venosa. A ocorrência de insuficiência venosa crônica e ulcerações estão relacionadas à presença do fator etiológico que é a insuficiência venosa desencadeada pela hipertensão venosa, com isso o paciente que apresenta DM tem a maior dificuldade de cicatrização das lesões. A Tabela 1 detalha dos dados coletados em prontuário: 
Tabela 1 - Amostra os dados coletados nos prontuários.

\begin{tabular}{cccccccc}
\hline Iniciais & Idade & Sexo & Estado Civil & Comorbidades & $\begin{array}{c}\text { Etiologia } \\
\text { da Ferida }\end{array}$ & IMC & Escolaridade \\
\hline JRV & 57 & M & Solteiro & DM & IV & 22,3 & $2^{\circ}$ grau completo \\
\hline LPS & 67 & F & Solteira & DM/HAS & IV & 24,6 & $\begin{array}{c}\text { Ensino } \\
\text { Fundamental }\end{array}$ \\
\hline NSR & 74 & M & Sollteiro & DM & IV & 22,1 & $\begin{array}{c}2^{\circ} \text { grau } \\
\text { incompleto }\end{array}$ \\
\hline ESP & 45 & F & Solteira & DM/ Obesidade & IV & 40,3 & $2^{\circ}$ grau completo \\
\hline CPA & 63 & M & Solteiro & $\begin{array}{c}\text { DM/HAS/ } \\
\text { Sobrepeso }\end{array}$ & IV & 28,6 & $2^{\circ}$ grau completo \\
\hline
\end{tabular}

Legenda: IMC: Índice de massa corporal; DM: Diabetes Mellitus; HAS: Hipertensão Arterial Sistêmica.

Fonte: Andrade RV, et al., 2019.

Analisando os resultados da tabela acima, a faixa etária variou entre 45 e 74 anos, sendo 3 pacientes com idade superior a 60 anos e 2 pacientes com idade inferior à 60 anos. Biologicamente, o avanço da idade é marcado por transformações fisiológicas nas células, epiderme, tecidos e orgãos que ocasionam consequências na capacidade funcional do idoso tornando-o mais predisposto às doenças crônicas (TAVARES APC, et al., 2017). Contudo, o envelhecimento contribue para as fragilidades da epiderme, propiciando aparecimento de lesões, úlceras e interferindo também no processo de cicatrização. Sendo esse fator percebido na amostra do estudo, no qual $3(60 \%)$ dos pacientes tem acima de 60 anos.

Foi identificada uma prevalência do sexo masculino em relação ao feminino nos nossos achados. Desse modo, estudos demonstram que o sexo masculino possui dificuldade em buscar atendimento de saúde para promoção e prevenção e somente procuram atendimento quando ocorrem complicações na saúde (SQUIZATTO RH, et al., 2017). Assim, percebe-se vulnerabilidade de comportamento e autoestima de homens acometidos por UV, impactanto nas relações sociais, sexuais e emocionais destes. Neste estudo foram referidos que $100 \% \%$ dos participantes são solteiros e a presença de um parceiro é condição importante no manejo da doença por parte de indivíduos diabéticos e portadores de úlceras venosas, uma vez que buscam incentivar os pacientes a aderirem ao tratamento, para controlar hábitos não saudáveis e adotar estilos de vida favoráveis (SALIN AB, et al., 2019).

Dentre as comorbidades, todos possuem DM, 2 possuem DM associado à Hipertenão Arterial Sistêmica (HAS), 1 possue DM e Obesidade e o outro possue DM/HAS e sobrepeso. Todos os pacientes possuem úlcera de membros inferiores de etiologia venosa. A DM acomete um menor aporte de oxigênio, destruição e má formação na migração de fibroblastos, o que demanda um cuidado específico no processo de avaliação. A HAS favorece a redução do oxigênio, modificações vasculares, essas duas atividades são dependentes, sendo assim, pacientes portadores desta comorbidade podem ter reparação tecidual insuficiente (MARQUES ADB, et al., 2015). Dessa forma, essas duas comorbidades interferem na evolução da cicatrização das feridas, pois interfere na perfusão dos tecidos e no aumento do risco de desenvolvimento de infecção no leito da lesão, sendo importante ressaltar a relevância do papel do enfermeiro desde o momento da anamnese, a sistematização da assistência, quanto as orientações fornecidas ao paciente sobre as suas comorbidades e o quanto o descontrole dessas doenças vão impactar no tratamento da lesão.

Assim, o DM tem relação com a precocidade da Doença Vascular, adiantando sua ocorrência em torno de 15 anos, nos indivíduos diabéticos não controlados. O trabalho de prevenção contra o DM ainda é o mais eficaz. Pode ocorrer de duas formas, contra o início do DM (prevenção primária) ou de suas complicações agudas ou crônicas (prevenção secundária) (FERREIRA DL, et al., 2019).Tais estratégias consistem em Educação em Saúde, grupos 
de orientação preventiva, reuniões com profissionais da saúde, entre outros, principalmente o enfermeiro, através da consulta de enfermagem consegue fazer uma anamnese e exame físico específico, acompanhado de promoção a saúde sobre os cuidados e a prevenção com as comorbidades.

A atenção primária com a prevenção e promoção a saúde, aonde investiga os fatores intrínsecos e extrínsecos que comprometem ao surgimento das úlceras venosas são primordiais na avaliação para esse cuidado. Dentre eles como os fatores intrínsecos, estes sendo as doenças crônicas como a DM e a Insuficiência Venosa (IV) além da idade avançada, falta de educação em saúde e maior números de comorbidades, ao colacionar aos extrínsecos como saneamento básico, melhoria da alimentação e condições de trabalhos, se depara com fatores de riscos importantes atenuados do surgimento dessas lesões venosas (TAVARES APC, et al., 2017). Com isso, os pacientes que com a doença do DM e IV são mais susceptíveis ao aparecimento dessas lesões.

Assim, ao questionar a etiologia da IV observou a prevalência de $100 \%$ em todos os pacientes participantes desse estudo.Dentre os 5 prontuários, 2 apresentam IMC dentro da normalidade, 2 pacientes possuem IMC alterado, 1 com sobrepeso e outro com obesidade e o paciente NSR não possui cálculo de IMC, pois não constava em prontuário o registro de peso e altura. $O$ aumento de peso e a obesidade influenciam de forma negativa no processo cicatricial, decorrente da má circulação que reduzem o aporte de oxigênio e nutrientes nos tecidos (NOGUEIRA GA, et al., 2015). Com isso, cabe ao enfermeiro orientar ao paciente sobre a importância da alimentação rica em nutrientes, proteínas e vitaminas, enfatizando que a deficiência nutricional acarreta no prejuízo de todo o processo cicatricial. O excesso de peso também é um fator que interfere na cicatrização e dificulta o tratamento dos pacientes acometidos pela UV, devido à diminuição do aporte sanguíneo para os tecidos, favorecendo o surgimento de lesões e infecções.

Quanto ao grau de escolaridade, 3 pacientes tinham $2^{\circ}$ grau completo, 1 paciente $2^{\circ}$ grau incompleto e 1 paciente possuia ensino fundamental incompleto, ou seja, os pacientes nesse estudo tem nível de entendimento que oscila entre bom e razoável. O nível de escolaridade é um fator relevante no processo de tratamento do paciente, pois pode ocorrer dificuldade em entender as informações fornecidas no processo do cuidado, sobre as orientações do autocuidado que ele deve ter em domicílio e tudo que for implementado na assistência de enfermagem. $O$ enfermeiro deve estar atento se a comunicação foi efetiva nessas orientações e o fazer compreender que ele é peça indispensável para que o tratamento seja eficaz. O nível inferior de escolaridade é um aspecto significativo na maneira como esse paciente conduz o seu autocuidado e o estilo de vida apropriado para sua situação, que decorre dos conhecimentos adquiridos durante sua vida (TAVARES APC, et al., 2017).

Desta forma, é importante que o enfermeiro esteja atento ao entendimento do paciente sobre as condutas de cuidado, utilizando de linguagem clara e confimando sempre se o paciente esta compreendendo as orientações, esclarecendo suas dúvidas, caso ocorra, procurando envolver também a família nesse processo de cuidado.

Dentre as funções desempenhadas pelo enfermeiro o processo de ensino é essencial para esses pacientes. Todas as orientações dadas durante o momento do cuidado e troca de curativo torna-se essencial para contribuir na cicatrização dessas lesões, bem como na prevenção de agravos relacionadas à patologia, devendo essa prevenção ocorrer por intermédio de aplicação de novas técnicas com intervenções de enfermagem para promover a recuperação e reabilitação não somente do processo cicatrical, visto que, diversos fatores interferem nesse processo, como dieta, controle de pressão arterial, glicemia descompensada, fatores externos que podem provocar agressão, além de outros fatores fisiológicos (JOAQUIM FL, et al., 2018).

Durante o tratamento das úlceras, que em sua grande maioria é lento, o paciente deve seguir atentamente as orientações realizadas pelo profissional, pois são essenciais para o sucesso desse tratamento (PIRES, JO, OLIVEIRA RF, CRUZ NR, 2016). Ademais, na caracteristica da UV, deve-se observar a localização, o tipo de tecido que é mais prevalente na lesão, o formato das bordas e integridade da área perilesional e os tipos e quantidades de exsudatos, como descreva abaixo na Tabela 2.

REAS/EJCH | Vol.Sup.n.48 | e3070 | DOI: https://doi.org/10.25248/reas.e3070.2020 Página 5 de 8 
Tabela 2 - Características clínicas da úlcera venosa

\begin{tabular}{|c|c|c|c|c|c|}
\hline Iniciais & Localizaçao & Tecido & Bordas & Área Perilesional & Exsudato \\
\hline JRV & $\begin{array}{l}\text { Maléolo } \\
\text { externo em } \\
\text { MID }\end{array}$ & 95\% granulação & $\begin{array}{l}\text { Irregulares e } \\
\text { secas }\end{array}$ & Hiperpigmentada & $\begin{array}{c}\text { Amarelado, de aparência } \\
\text { purulenta, odor intenso e } \\
\text { pequena drenagem }\end{array}$ \\
\hline LPS & $\begin{array}{l}\text { Malélo } \\
\text { interno em } \\
\text { MIE }\end{array}$ & $30 \%$ granulação & $\begin{array}{l}\text { Irregulares e } \\
\text { secas }\end{array}$ & Hiperpigmentada & $\begin{array}{c}\text { Amarelado, de aparência } \\
\text { purulenta, odor discreto e } \\
\text { pequena drenagem }\end{array}$ \\
\hline NSR & $\begin{array}{l}\text { Maléolo } \\
\text { externo em } \\
\text { MID }\end{array}$ & $20 \%$ granulação & $\begin{array}{l}\text { Regulares e } \\
\text { maceradas }\end{array}$ & $\begin{array}{l}\text { Hiperpigmentada } \\
\text { e ressecada }\end{array}$ & $\begin{array}{c}\text { Amarelado, de aparência } \\
\text { purulenta, inodoro e } \\
\text { moderada drenagem }\end{array}$ \\
\hline ESP & $\begin{array}{l}\text { Tibial lateral } \\
\text { em MID }\end{array}$ & $80 \%$ granulação & $\begin{array}{l}\text { Irregulares e } \\
\text { maceradas }\end{array}$ & $\begin{array}{l}\text { Hiperpigmentada, } \\
\text { edemaciada e } \\
\text { hiperemiada }\end{array}$ & $\begin{array}{l}\text { Amarelado, de aparência } \\
\text { purulenta, inodoro e } \\
\text { pequena drenagem }\end{array}$ \\
\hline CPA & MID & $\begin{array}{l}\text { Presença de } \\
\text { tecido de } \\
\text { granulação e } \\
\text { esfacelo }\end{array}$ & $\begin{array}{l}\text { Irregulares e } \\
\text { maceradas }\end{array}$ & Hiperpigmentada & $\begin{array}{l}\text { Amarelado, de aparência } \\
\text { purulenta, inodoro e } \\
\text { pequena drenagem }\end{array}$ \\
\hline
\end{tabular}

Fonte: Andrade RV, et al., 2019.

Nessa segunda tabela avaliou-se as características das feridas. O primeiro tópico analisado é a localização. A região que mais atinge o portador é a porção distal dos membros inferiores, sendo predominante a região maleolar (OLIVEIRA BGRB, et al., 2012). Sendo assim, das lesões analisadas nos prontuários, $60 \%$ estão localizadas na região do maléolo nos membros inferiores e $40 \%$ na região tibial corroborando assim, uma característica da úlcera venosa.

Em relação ao tipo de tecido presente na lesão, todos apresentavam variação entre tecido de granulação e esfacelo, $2 \mathrm{com}$ maior presença de tecido de granulação, 2 pacientes com presença de esfacelo no leito da ferida e um paciente que no prontuário não constava o percentual. A maior prevalência de tecido de granulação no leito das feridas nos remete a uma boa evolução, pois o próximo estágio, a epitelização, que é o ínicio da oclusão, necessita que a lesão seja preenchida por esse tecido. $O$ esfacelo presente no leito da ferida evidencia que a cicatrização está prejudicada (RIBEIRO APL e OLIVEIRA BGRB, 2015).

Bordas maceradas impedem o deslocamento dos queratinócitos para o centro da lesão, impedindo que o aparecimento do tecido de epitelização. A maceração pode ocorrer quanto ao uso estendido ou errado de coberturas que promovem o excesso de exsudato nas feridas (OLIVEIRA BGRB, et al., 2012). Nos dados coletados houve uma prevalência de pacientes com bordas maceradas o que requer do enfermeiro uma alerta nesse sinal, pois, é a partir da contração das bordas que ocorre o processo de cicatrização. Sendo assim, é importante que o enfermeiro utilize do seu conhecimento científico através de cuidados específicos e coberturas que ofereçam proteção a essa borda, bem como trata-las de forma individual.

O exsudato é um sinal relevante na avaliação da ferida, por acometer o processo cicatricial e ser um sinal sugestivo de infecção interferindo assim, nas fases de cicatrização (BUDÓ MLD, et al., 2015). As características analisadas em relação ao exsudato nos pacientes selecionados foram predominantemente de cor amarelada, com aparência purulenta, pequena drenagem e inodoro. Essas características reforçam a importância do cuidado da enfermagem, em que o enfermeiro deve ser capaz de avaliar essas especificidades, tendo conhecimento teórico sobre todos os processos de cicatrização e avaliação da ferida, utilizando coberturas e produtos adequados para 
a evolução das lesões. Torna-se necessário que o enfermeiro esteja sempre atualizado sobre o surgimento de novas coberturas, a fim de oferecer um melhor tratamento para o paciente, adequando sempre ao seu nível socieconômico (SILVA ACO, et al., 2017).

O paciente deve ser orientado a importância do acompanhamento da evolução da lesão e a necessidade da troca periódica do curativo mediante as orientações do enfermeiro, pois este também é um fator que contribui para o retardo no processo de cicatrização, favorecendo o aparecimento de infecções, aumentando a incidência de odor da ferida e causando também desconforto e diminuição da qualidade de vida do paciente (FREDERICO GA, et al., 2018).

A área perilesional hiperpigmentada é um sinal da úlcera venosa que acontece quando há extravasamento da hemoglobina para o interstício, com isso a homossiderina é liberada e o pigmento da mesma adere aos tecidos com uma coloração meio azulada (OLIVEIRA BGRB, et al., 2012). A ocorrência de descamação e ressecamento da pele também é uma característica das úlceras venosas. Essas características contribuem para o surgimento de novas lesões, e até mesmo para o agravamento da lesão já existente. O enfermeiro deve estar atento também a essas características e observar com cuidado essa a área, utilizando produtos que permitam a hidratação dessa área evitando que novas lesões ocorram (CAMPO ALM, et al., 2019).

Quanto aos cuidados no tratamento de UV, é importante ressaltar que estratégias para um bom retorno venoso devem ser prioridade. Elevar os membros acima da linha cardíaca, utilizar meias de compressão e exercícios que estimulem o músculo gastrocnêmio, são medidas que vão aliviar as dores, reduzir o edema e vão impactar de forma considerável o retorno venoso (SOUSA HF, et al., 2015). Todas essas orientações devem ser transmitidas para o paciente e reforçadas ao familiar que o acompanhe, pois são cuidados que tem grande importância no processo de evolução da UV. Para além de todos os cuidados físicos que esse paciente necessita, o portador de UV possue várias complicações que vão impactar nos hábito e qualidade de vida do mesmo (TAVARES APC, et al., 2017). Bem como prejuízos que implicam em afastamento social, depressão, diminuição da auto estima, desligamento do trabalho e idas com frequência ao ambulatório (SHIRLEI AF, 2018). Desse modo, é imprescindível que o profissional de saúde esteja voltado para as modificações biopsicossocias desencadeadas pela UV (TAVARES APC, et al., 2017).

Ademais, percebe-se limitações do estudo principalmente, de ordem física, haja visto que os prontuários eram registrados por estudantes sob supervisão da preceptoria, entretanto alguns com letras ilegíveis ou incompreensíveis, assim como ausência de dados complementares relevantes que foram subnotificados e poderiam ter ajudado com outras informações importantes.

\section{CONCLUSÃO}

Assim, para que a assistência seja prestada de forma correta, além da interação do paciente portador de UV no processo de cicatrização, o enfermeiro precisa de uma educação adequada, capacitação, habilidades e competências, utilizando essas questões aplicadas á real vivencia tanto do profissional como do paciente. Utilizando de presuposto que o enfermeiro diante esse cenário do cuidado a pele dos pacientes, tem uma grande relevância visto são eles que passa um maior e melhor tempo com o paciente onde isto proporciona um contato que é eficaz e eficiente para o desenvolver do tratamento, acompanha a evolução da ferida devido ter a formação voltada para este tipo de prática. É possivel concluir através deste estudo que o papel do enfermeiro no processo de cicatrizaçao e avaliação das feridas em pacientes portadores DM E IV é de fundamental importância visto que o enfermeiro avalia toda lesão e faz planejamento do cuidado com intervenções adequada a cada tipo de ferida. Alé do cuidado com a lesão, percebe-se que o enfermeiro junto à equipe multidiciplinar deve criar estratégias que proporcione uma qualidade de vida melhor, condições que minimizem o tempo de cicatrização da ferida, a dor, os riscos de infecções, o impacto da lesão na sua auto estima e a prevenção de recidivas. Esses pacientes são propensos a desenvolver depressão e baixa autoestima, o que dificulta o processo do cuidados, levando ao isolamento social.

REAS/EJCH | Vol.Sup.n.48 | e3070 | DOI: https://doi.org/10.25248/reas.e3070.2020 Página 7 de 8 


\section{REFERÊNCIAS}

1. BORGE EL, et al. Prevenção de recidiva de úlcera varicosa: um estudo de coorte. Acta Paul Enferm. 2016; 29(1): 9-16.

2. BUDÓ MLD, et al. Úlcera venosa, índice tornozelo braço e dor nas pessoas com úlcera venosa em assistência no ambulatório de angiologia. R. Enferm. Cent. O. Min, 2015; (3): 1794-1804.

3. CAMPO ALM, et al. Assistência de enfermagem a pacientes com feridas crônicas: um relato de experiência. REFACS (online), 2019; 7(2): 248-255.

4. DIAS TYAF, et al. Avaliação da qualidade de vida de pacientes com e sem úlcera venosa. Rev. Latino-Am. Enfermagem, 2014; 22(4): 576-81.

5. Diretrizes da Sociedade Brasileira de Diabetes 2017-2018. Classificação e diagnóstico do diabetes mellitus. Disponível em: Pág. 18, 19, 20.

6. FERREIRA DL, et al. Efeito das equipes multiprofissionais em saúde no brasil em atividades de cuidado com o diabetes. Revista Eletrônica Acervo Saúde. 2019; S17: e91.

7. FREDERICO GA, et al. Integralidade no cuidado de enfermagem às pessoas com úlceras Cutâneas. Rev enferm UFPE on line., 2018; 12(7): 1997-2011.

8. JOAQUIM FL, et al. Impacto das úlceras venosas na qualidade de vida dos pacientes: revisão integrativa Rev Bras Enferm [Internet], 2018; 71(4): 2137-46.

9. JOAQUIM FL, et al. Repercussão da visita domiciliar na capacidade funcional de pacientes com úlceras venosas Rev Bras Enferm [Internet], 2017; 70(2): 304-11.

10. LEAL TS, et al. Percepção de Pessoas com Ferida Crônica. Rev enferm UFPE online. Recife, 2017; 11(3): 1156-62.

11. MARQUES ADB, et al. Critérios utilizados pelos enfermeiros na realização dos curativos. Rev. Pre. Infec e Saúde, 2015; 1(1): 31-39.

12. NOGUEIRA GA, et al. Diagnósticos de enfermagem em pacientes com úlcera venosa crônica: estudo observacional Rev. Eletr. Enf. [Internet], 2015; 17(2): 333-9.

13. OLIVEIRA BGRB, et al. Caracterização dos pacientes com úlcera venosa acompanhados no Ambulatório de Reparo de Feridas. Rev. Eletr. Enf. [Internet], 2012; 14(1): 156-63.

14. PIRES JO, et al. Assistência de enfermagem no controle e manejo da úlcera venosa. Revista Transformar, №8, 2016.

15. RIBEIRO APL, et al. Efetividade dos géis de papaína a $2 \%$ e $4 \%$ na cicatrização de úlceras venosas* Rev Esc Enferm USP, 2015; 49(3): 395-402.

16. SALVETTI MG, et al. Prevalência de dor e fatores associados em pacientes com úlcera venosa. Rev Dor. São Paulo, 2014; 15(1): 17-20

17. SHIRLEI AF. Cuidado do enfermeiro junto à pessoa com úlcera venosa. Artigo científico apresentado à Disciplina de TCC II do curso de Enfermagem da Universidade Católica do Salvador, 2018.

18. SILVA ACO, et al. As principais coberturas utilizadas pelo enfermeiro.Revista Uningá, 2017; 53(2): 117-123.

19. SALIN AB, et al. Diabetes Mellitus tipo 2: perfil populacional e fatores associados à adesão terapêutica em Unidades Básicas de Saúde em Porto Velho - RO. Revista Eletrônica Acervo Saúde. 2019; S33: e1257.

20. SOUSA HF, et al. O enfermeiro no manejo clínico de pacientes com úlcera venosa: revisão integrativa de literatura. Revista Humano Ser - UNIFACEX, Natal-RN, 2015; 1(1): 32-51.

21. SQUIZATTO RH, et al. Perfil dos usuários atendidos em ambulatório de cuidado com feridas. Pág 6 Cogitare Enferm. 2017; 22(1): 01-09.

22. TAVARES APC, et al. Qualidade de Vida de Idosos Pág. 2. Esc Anna Nery 2017;21(4): e20170134PILGER C, et al. Características sociodemográficas e de saúde de idosos: contribuições para os serviços de saúde. Rev. Latino-Am. Enfermagem Rev Latino-Am. Enfermagem, 2011; 19(5): 1230-1238.

23. TAVARES BC, et al. Perfil dos pacientes com úlcera crônica de etiologia diversa, internados em serviço de emergência. Cienc Cuid Saude, 2016; 15(4): 624-629. 